\title{
COVID-19 and Arrhythmias: A Review of Cardiac Electrophysiology Associated with SARS-CoV-2
}

Kashmala Khan, MD, Francis Dimtri, DO, Carlos Vargas, DO,' Thomas Alexander, MD ${ }^{1}$

\section{Abstract}

\section{Description}

Coronavirus infectious disease 2019 (COVID-19) has become one of the most recognized viral illnesses to date. Most people infected with the severe acute respiratory syndrome coronavirus-2 (SARS-CoV-2) have a benign clinical course, but there have also been catastrophic outcomes. Although, notoriously known to affect the respiratory system, extra-pulmonary manifestations have also been established. An increasing number of people have been reported to present with cardiac manifestations, which include but are not limited to arrhythmias. The cardiac conduction system can be damaged by various mechanisms in patients infected with SARS-CoV-2. In this review, we will attempt to briefly describe the arrhythmogenic potential of SARS-CoV-2 and the factors that play a key role in the electrophysiological pathology seen in COVID-19.

\section{Keywords}

SARS-CoV-2; COVID-19; coronavirus infections/complications; cardiac arrhythmias; cardiac arrhythmias/epidemiology; pandemics; viral pneumonia; hypokalemia; prolonged QT; EKG abnormalities; myocardial injuries

\section{Introduction and Background}

Cardiac manifestations of severe acute respiratory syndrome coronavirus-2 (SARS-CoV-2) infection have been well established in recent literature. One study has shown patients with underlying cardiovascular disease who are infected with coronavirus disease 2019 (COVID-19) have a $10.5 \%$ fatality rate. ${ }^{1}$ Cardiac injury or damage has been observed in patients with COVID-19. In a retrospective study done by Zhou et al., patients infected with SARSCoV-2 were found to have various cardiac manifestations. Heart failure was the third most common (23\%) observed complication in the study population. Cardiac sensitive troponin I was found in more than half of the patients who did not survive. ${ }^{2}$ A retrospective observational study conducted by Yang and colleagues found that cardiac injury (defined as elevated troponin I levels) was present in $23 \%$ of patients. ${ }^{3}$ Another study exhibited that among 138 hospitalized patients infected with COVID-19, $16.7 \%$ developed arrhythmias. ${ }^{4}$ In another ret- rospective multicenter study, $40 \%$ of fatalities were attributed to myocardial injury. ${ }^{5}$ Finally, a study conducted by Du et al. determined that $8.6 \%$ of deaths in the study population were from cardiac arrest. ${ }^{6}$ Thus, infection with SARS-CoV-2 is associated with cardiac injury, which may manifest as arrhythmias. (Figure 1)

\section{Review}

Damage to the myocardium may manifest as conduction system damage, tachyarrhythmias, bradyarrhythmias, drug-induced QT prolongation and sudden cardiac death have been described within the literature. Arrhythmias seen with COVID-19 include sinus tachycardia, sinus bradycardia, atrioventricular block, bundle branch block, atrial fibrillation, supraventricular tachycardia, torsade des pointes, non-sustained ventricular tachycardia, sustained ventricular tachycardia, ventricular fibrillation and pulseless electrical activity. ${ }^{7}$ A recent cross-sectional survey was sent to more than 1,100 electrophysiologists worldwide to evaluate the ar-

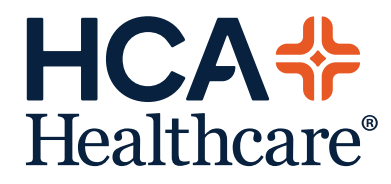

www.hcahealthcarejournal.com

(c) 2020 HCA Physician Services, Inc. d/b/a Emerald Medical Education
HCA Healthcare
Journal of Medicine 


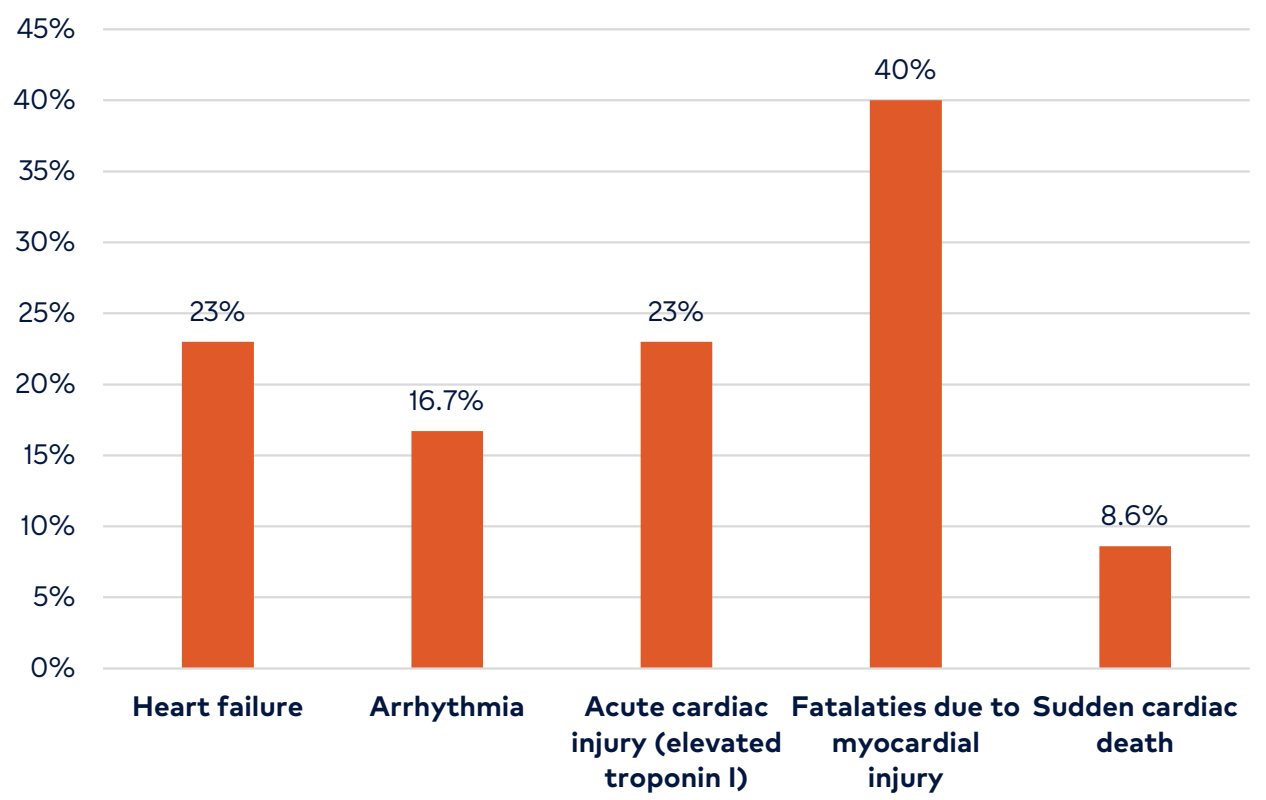

Figure 1. Graph depicting the incidence of cardiac manifestations seen in COVID-19, as per multiple studies.1-6

rhythmic manifestations of COVID-19. According to the results of the survey, severe sinus bradycardia and complete heart block were the most commonly reported bradyarrhythmia and atrial fibrillation as the most common tachyarrhythmia reported amongst hospitalized patients with COVID-19.8

Most of the arrhythmias that have been documented have occurred in the hospital setting. Wang et al. demonstrated that in hospitalized patients with COVID-19, 16.7\% patients had a documented arrhythmia and of those $44 \%$ were admitted in the intensive care unit (ICU). ${ }^{4}$ A cohort study done in New York established that atrial arrhythmias were seen in $17.7 \%$ of patients requiring mechanical ventilation and only $1.9 \%$ of patients on non-invasive ventilation. ${ }^{9}$ Bhatla et al. conducted a cohort study to evaluate the incidence of cardiac arrest and various arrhythmias in hospitalized patients. This study exhibited that a higher incidence of cardiac arrest and arrhythmias was seen in patients admitted to the ICU when compared to the non-ICU population. ${ }^{10}$

Arrhythmias have become a well-recognized manifestation of COVID-19. Several theories exist as to why cardiac arrhythmias are present in COVID-19 patients. (Figure 2) First, mechanisms of arrythmias include direct myocardial damage, which can directly damage myocardial cells by setting up an inflammatory response. After entering the myocardium, the virus proliferates, causing oxidative stress and direct injury to the cardiac myocytes. An inflammatory response is then initiated by the host. The host response includes $T$ cell activation, cytokine release and antibody formation. This leads to interstitial edema, endothelial dysfunction and cardiac fibrosis. Cardiac remodeling and dilatation is seen, which leads to an impaired myocardium. Acute viral myocarditis and pericarditis occur as a result and can be self-limiting with minimal symptoms, but occasionally, atrial or ventricular arrhythmias can be seen as a consequence. ${ }^{11,12}$

Second, hypoxia, a known complication of COVID-19 infection, is also a well-recognized risk factor for arrhythmias. Given the immense pulmonary involvement of SARS-CoV-2, hypoxia is one of the major sequelae seen in COVID-19. Acute lung injury can lead to hypoxemia, which can trigger instability in the myocardial electrical balance. As a result, decreased oxygen delivery can alter the action potential and has contributed to arrhythmias encountered during this pandemic.13,14

Next, the host immune response to the virus itself may also contribute to arrhythmogenesis. Inflammatory mediator release of interleukin-1 (IL-1), IL-6, IL-7, interferon-gamma and tumor 


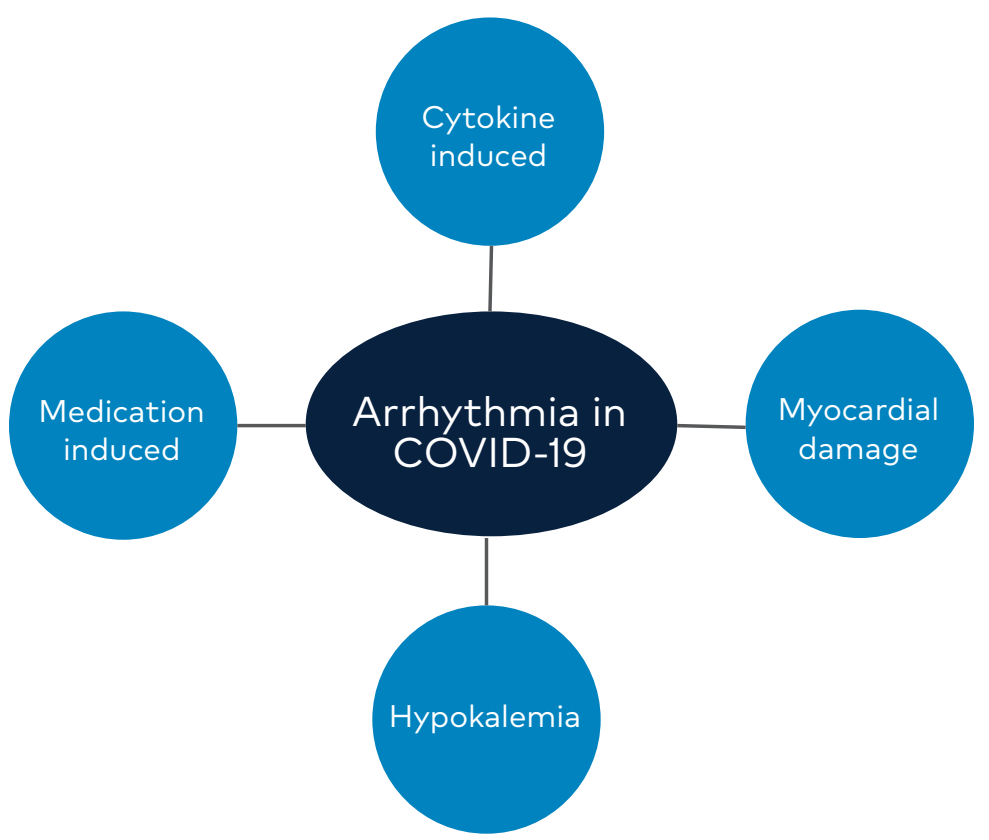

Figure 2. Various mechanisms implicated in causing arrhythmias in COVID-19.

necrosis-alpha are postulated to adversely affect cardiac repolarization. These inflammatory mediators may inhibit a cardiac ion channel protein of the delayed rectified current, Ikr. The functional blockade of Ikr may lead to delayed repolarization and subsequent prolonged QT on electrocardiogram (ECG). ${ }^{15,16}$

Furthermore, electrolyte derangement, specifically hypokalemia, is associated with SARSCoV-2 infection. As electrolyte imbalances, specifically hypokalemia, have been proven to cause arrhythmias, patients with COVID-19 potentially can be more susceptible to developing arrhythmias. The virus enters the host via the ACE2 receptor, which can alter the renin-angiotensin-aldosterone system (RAAS). The virus may degrade the ACE2 receptors, disrupting their ability to regulate the RAAS, leading to electrolyte imbalances. Another source of hypokalemia in these patients is due to gastrointestinal symptoms (diarrhea and vomiting). ${ }^{17,18}$ In the cohort study conducted by Chen et al., hypokalemia was seen in $85 \%$ of patients who were critically ill with COVID-19.8 Close monitoring on telemetry is advisable, as is consulting a cardiologist for these patients earlier in the course of the disease. It is also important to note that over-correction of electrolytes can also precipitate certain arrhythmias.

Finally, medications used in the management of SARS-CoV-2 can also lead to arrhythmias. While treating COVID-19 effectively is important, physicians and other medical providers must ensure that therapeutic agents are renally dosed and drug-drug interactions that potentiate QT-prolongation are eliminated. Drugs that are used include, anti-virals, immunosuppressants, anti-arrhythmics, antibiotics, anti-psychotics and anti-emetics. The use of these medications (alone or in combination) can cause prolonged QT, torsades de pointes, atrioventricular block, supraventricular tachycardia and bradycardia. ${ }^{19,20}$ Medications should also be renally dosed for patients with renal insufficiency. A detailed list of medications and their respective cardiac manifestations are provided in Table 1.

\section{Conclusion}

COVID-19 has been shown to be pro-arrhythmogenic, and arrhythmias are underappreciated as a cause of increased morbidity and mortality. Infection results in inflammatory conditions that may further precipitate arrhythmias. Health care providers should be cognizant of monitoring with telemetry those at highest risks for arrhythmias, which would include patients with cardiac injury (patients with elevated troponin), those who have structural heart disease and known history of arrhythmias. Prevention includes obtaining baseline ECG while monitoring with telemetry, 
Table 1. A list of some drugs used in the management of COVID-19 and their respective cardiac manifestations. ${ }^{19,20}$

\begin{tabular}{ccc} 
Drug Name & Drug Class & Possible Cardiac Side Effect \\
\hline Amiodarone & Anti-arrhythmic & QT prolongation \\
\hline Flecainide & Anti-arrhythmic & QT prolongation \\
\hline lbutilide & Anti-arrhythmic & QT prolongation \\
\hline Procainamide & Anti-arrhythmic & QT prolongation \\
\hline Quinidine & Anti-arrhythmic & QT prolongation \\
\hline Sotalol & Anti-arrhythmic & QT prolongation \\
\hline Azithromycin & Antibiotic & QT prolongation \\
\hline Levofloxacin & Antibiotic & QT prolongation \\
\hline Fluconazole & Anti-fungal & QT prolongation \\
\hline Domeperidone & Anti-emetic & QT prolongation \\
\hline Odansetron & Anti-emetic & QT prolongation \\
\hline Haloperidol & Anti-psychotic & QT prolongation \\
\hline Resperidone & Anti-psychotic & QT prolongation \\
\hline Chloroquine & Anti-malarial & QT prolongation \\
\hline Hydroxychloroquine & Anti-malarial & QT prolongation \\
\hline Propofol & Anesthetic & QT prolongation \\
\hline Ribavirin & Anti-viral & Tachycardia, MI, Cardiomyopathy, arryhtmia \\
\hline Lopinavir/Ritonavir & Anti-viral & Ischemia Events, Atrioventricular block \\
\hline Methyprednisone & Steroid & Electrolyte imbalances \\
\hline Tocilizumab, Eculizumab & Immunosuppressants & Bradycardia, Atrioventricular block \\
\hline Bevacizumab & Immunosuppressants & Congestive heart failure, Supraventricular \\
\hline thith & & tachycardia \\
\hline
\end{tabular}

with emphasis on QTc or AV block, avoiding QT prolonging medications, reducting inflammatory response and consulting electrophysiology early, when necessary. High-risk patients should practice social distancing and be cautious of potential QT prolonging medications, consulting their physician when using them.

\section{Conflicts of Interest}

The authors declare they have no conflicts of interest.

The authors are employees of Corpus Christi Medical Center, a hospital affiliated with the journal's publisher.

This research was supported (in whole or in part) by HCA Healthcare and/or an HCA Healthcare affiliated entity. The views expressed in this publication represent those of the author(s) and do not necessarily represent the official views of HCA Healthcare or any of its affiliated entities.

\section{Author Affiliations}

1. Corpus Christi Medical Center, Corpus Christi, TX

\section{References}

1. American College of Cardiology. ACC clinical bulletin: COVID-19 Clinical Guidance for the Cardiovascular Care Team. https://www.acc.org// / media/Non-Clinical/Files-PDFs-Excel-MSWord-etc/2020/02/S20028-ACC-Clinical-Bulletin-Coronavirus.pdf. Accessed Feb 2020.

2. Zhou F, Yu T, Du R, et al. Clinical course and risk factors for mortality of adult inpatients with COVID-19 in Wuhan, China: a retrospective cohort study. Lancet. 2020;395(10229):1054-1062. https://doi.org/10.1016/s0140-6736(20)30566-3

3. Yang $X, Y u Y, X \cup J$, et al. Clinical course and outcomes of critically ill patients with SARS-CoV-2 pneumonia in Wuhan, China: a single-centered, 
retrospective, observational study. Lancet Respir Med. 2020;8(5):475-481. https://doi.org/10.1016/ s2213-2600(20)30079-5

4. Wang D, Hu B, Hu C, et al. Clinical Characteristics of 138 Hospitalized Patients With 2019 Novel Coronavirus-Infected Pneumonia in Wuhan, China. JAMA. 2020;323(11):1061-1069. https://doi. org/10.1001/jama.2020.1585

5. Ruan Q, Yang K, Wang W, Jiang L, Song J. Clinical predictors of mortality due to COVID-19 based on an analysis of data of 150 patients from Wuhan, China [published correction appears in Intensive Care Med. 2020 Apr 6]. Intensive Care Med. 2020;46(5):846-848. https://doi. org/10.1007/s00134-020-05991-x

6. Du Y, Tu L, Zhu P, et al. Clinical Features of 85 Fatal Cases of COVID-19 from Wuhan. A Retrospective Observational Study. Am J Respir Crit Care Med. 2020;201(11):1372-1379. https://doi. org/10.1164/rccm.202003-0543oc

7. Manolis AS, Manolis AA, Manolis TA, Apostolopoulos EJ, Papatheou D, Melita H. COVID-19 infection and cardiac arrhythmias [published online ahead of print, 2020 Aug 16]. Trends Cardiovasc Med. 2020;S1050-1738(20)30107-9. https:// doi.org/10.1016/j.tcm.2020.08.002

8. Gopinathannair R, Merchant FM, Lakkireddy DR, et al. COVID-19 and cardiac arrhythmias: a global perspective on arrhythmia characteristics and management strategies. J Interv Card Electrophysiol. 2020;59(2):329-336. https://doi. org/10.1007/s10840-020-00789-9

9. Goyal P, Choi JJ, Pinheiro LC, et al. Clinical Characteristics of Covid-19 in New York City. N Engl J Med. 2020;382(24):2372-2374. https://doi. org/10.1056/nejmc2010419

10. Bhatla A, Mayer MM, Adusumalli S, et al. COVID-19 and cardiac arrhythmias. Heart Rhythm. 2020;17(9):1439-1444. https://doi. org/10.1016/j.hrthm.2020.06.016

11. Tse G, Yeo JM, Chan YW, Lai ET, Yan BP. What Is the Arrhythmic Substrate in Viral Myocarditis? Insights from Clinical and Animal Studies. Front Physiol. 2016;7:308. Published 2016 Jul 21. https:// doi.org/10.3389/fphys.2016.00308

12. Babapoor-Farrokhran S, Rasekhi RT, Gill D, Babapoor S, Amanullah A. Arrhythmia in COVID-19 [published online ahead of print, 2020 Aug 14]. SN Compr Clin Med. 2020;1-6. https://doi. org/10.1007/s42399-020-00454-2

13. Kochi AN, Tagliari AP, Forleo GB, Fassini GM, Tondo C. Cardiac and arrhythmic complications in patients with COVID-19. J Cardiovasc Electrophysiol. 2020;31(5):1003-1008. https://doi. org/10.1111/jce.14479

14. Macdonald WA, Hool LC. The effect of acute hypoxia on excitability in the heart and the L-type calcium channel as a therapeutic target. Curr Drug Discov Technol. 2008;5(4):302-311. https:// doi.org/10.2174/157016308786733546
15. Mehta P, McAuley DF, Brown M, et al. COVID-19: consider cytokine storm syndromes and immunosuppression. Lancet. 2020;395(10229):10331034. https://doi.org/10.1016/s01406736(20)30628-0

16. Aromolaran AS, Srivastava U, Alí A, et al. Interleukin- 6 inhibition of hERG underlies risk for acquired long QT in cardiac and systemic inflammation. PLoS One. 2018;13(12):e0208321. Published 2018 Dec 6. https://doi.org/10.1371/ journal.pone.0208321

17. Cooper LT Jr. Myocarditis. N Engl J Med. 2009;360(15):1526-1538. https://doi.org/10.1056/ nejmra0800028

18. Chen D, Li X, Song Q, et al. Assessment of Hypokalemia and Clinical Characteristics in Patients With Coronavirus Disease 2019 in Wenzhou, China. JAMA Netw Open. 2020;3(6):e2011122. Published 2020 Jun 1. https:// doi.org/10.1001/jamanetworkopen.2020.11122

19. Carpenter A, Chambers OJ, El Harchi A, et al. COVID-19 Management and Arrhythmia: Risks and Challenges for Clinicians Treating Patients Affected by SARS-CoV-2. Front Cardiovasc Med. 2020;7:85. Published 2020 May 5. https://doi. org/10.3389/fcvm.2020.00085

20. Borba MGS, Val FFA, Sampaio VS, et al. Effect of High vs Low Doses of Chloroquine Diphosphate as Adjunctive Therapy for Patients Hospitalized with Severe Acute Respiratory Syndrome Coronavirus 2 (SARS-CoV-2) Infection: A Randomized Clinical Trial. JAMA Netw Open. 2020;3(4):e208857. Published 2020 Apr 24. https://doi.org/10.1001/jamanetworkopen.2020.8857 